$$
\text { CONF-8308/32- } 2
$$

\title{
THEORY OF ELEMENTARY EXCITATIONS IN INTERMEDIATE VALENCE MATERIALS*
}

\author{
A. J. Fedro ${ }^{\star *}$ and S. K. Sinhat \\ Materials Science and Technology Division \\ Argonne National Laboratory, Argonne, Illinois
}

60439

October 1983

ing

The submitted manuseript has been authored
by a contractor of the U. S. Government
under contract No. W-31-109-ENG-3B.
Accordinglv, the U.S. Government retains a
nonexclusive, rovalty-free license to publish
or reproduce the published form of this
contribution, or allow cthers to da so, for
U.S. Government purposes.

$\operatorname{COTS}-8308132--2$

CEO4 003705

Manuscript submitted to "Moment Formation in Solids", 1983 NATO/CAP Institute, August 22-September 2, 19183, Victoria, British Columbia.

\section{DISCLAIMER}

\begin{abstract}
This report was prepared as an account of work sponsored by an agency of the United States Government. Neither the United States Government nor any agency thereof, nor any of their employees, makes any warranty, express or implied, or assumes any legal liability or responsibility for the accuracy, completeness, or usefulness of any information, apparatus, product, or process disclosed, or represents that its use would not infringe privately owned rights. Reference herein to any specific commercial product, process, or service by trade name, trademark, manufacturer, or otherwise does not necessarily constitute or imply its endorsement, recommendation, or favoring by the United States Government or any agency thereof. The views and opinions of authors expressed herein do not necessarily state or reflect those of the United States Government or any agency thereof.
\end{abstract} * Wor

supported by the U.S. Department of Energy. ** on

On leave from Northern Illinois University, DeKalb, IL 60115

$\dagger_{\text {Present address: Exxon Research and Engineering Company, Corporate Research }}$ Labs. Annandale, NJ 08801<smiles>CCC(C)CC(C)CCC(C)C</smiles> 
THEORY OF ELEMENTARY EXCITATIONS IA INTEPAEDIATE VALEIICE MATERIALS

\author{
A. J. Fedro** and S. K. Sinha ${ }^{+}$ \\ Materials Science and Technology Division \\ Argonne National Laboratory, Argonne, Illinois 60439
}

\begin{abstract}
We review the formalism for calculating the properties of systems represented by an Anderson lattice Hamiltonian. First, a nean-field theory for the one electron Green's function is presented and then extended to include spin and charge fluctuations which lead to a many-body resonance near the Fersi level. The dynamical spin susceptibility and neutron scattering cross section are also evaluated. Finally, we present a calculation which indicates the possibility of a Cooper-pairing mechanism induced by electron correlations.
\end{abstract}

\title{
1. Introduction
}

In these lectures, we will present various levels of a theoretical franework to understand the themodynamics and elementary excitations of a model system involving strong intrasite electron correlations, with a view to understanding the properties of the Anderson and Kondo lattices. First, we present the underlying operator formalism and indicate how it may be applied to obtain the simplest nean-field type theory for the Anderson lattice. Then, we show how the formalism may be extended to calculate the dynamical susceptibility, and also to the Kondo regime. Finally, we shall present some results relating to Cooper pairing of the electrons, driven by strong correlation effects, as a possible new mechanism for superconductivity in such materials.

Our starting point is the assumption that strong coulomb correlations between $f$-electrons have the result that all f-shell configurations other than $f^{n}$ and $f^{n-1}$ are projected out. Tnia is equivalent to stating that $\left.U_{f f}\right\}^{4}$. For simplicity, consider the case where $n=1$, and let $|i, 0\rangle$ denote the state at site $i$ where there are no 
f-electrons (or a full multiplet) and $|i, m\rangle$ denote the state rorresponding to the $\mathrm{m}^{\mathrm{th}}$ state of the multiplet corresponding to 'se $f-$ electron (or $f$-hole). (The states may equally well be crystalfield-split states.) We define operators (analogous to the standard basis operators ${ }^{1}$ ),

$$
f_{i m}^{+} \Delta|i, m\rangle\left\langle i, o\left|; f_{i n}=\right| i, o\right\rangle\langle i, m| \text {. }
$$

It is obvious from (1.1) that

$$
f_{i m}^{+} f_{i m^{\prime}}^{+}=0
$$

for all $\mathrm{m}, \mathrm{m}^{\prime}$, and the completeness property requires that

$$
|i, o\rangle\langle i, o|+\ldots| i, m\rangle\langle i, m|=1
$$

for each site $i$. Such operators, however, do not obey the usual Fermion anticommutation rules, but satisfy

$$
\begin{aligned}
& {\left[f_{i m^{+}}^{+}, f_{j m^{\prime}}^{+}\right]_{+}=\left[f_{i m}, f_{j m^{\prime}}\right]_{+}=0 ;} \\
& {\left[f_{i m^{\prime}}, f_{j m^{\prime}}^{+}\right]_{+}=\delta_{i j} Q_{m m^{\prime}}^{i},}
\end{aligned}
$$

where

$$
Q_{m m^{\prime}}^{i} \equiv \delta_{m m^{\prime}}\left(1-\sum_{m^{\prime \prime}} f_{i m^{\prime \prime}}^{+} f_{i m^{\prime \prime}}\right)+f_{i m^{\prime}}^{+} f_{i m} .
$$

Note that the expectation value $\left\langle Q_{\mathrm{mm}^{\prime}}^{i}\right\rangle$ is of $O\left(\frac{1}{N}\right)$ vinere $N$ is the number of states in the multiplet. We now write down the Hamiltonian for the Anderson lattice in the form

$$
\begin{aligned}
H & =\sum_{i, m} \varepsilon_{f m} f_{i m}^{+} f_{i m}+\sum_{k \sigma} c_{k \sigma} c_{k \sigma}^{+} c_{k \sigma} \\
& +\sum_{k \sigma}\left(N_{\ell}\right)^{-1 / 2} v_{k \sigma}^{m}\left[f_{i m}^{+} c_{k \sigma} e^{-i \vec{k} \cdot r_{i}}+h \cdot c \cdot\right] \\
& +\sum_{i, \mathbb{m}} e_{m} f_{i m}^{+} f_{i m} \delta V \\
& +\frac{1}{2} B(V) \delta V^{2}-g_{J} H_{B}^{H I} \sum_{i, m}\left\langle m\left|J_{z}\right| m\right\rangle f_{i m}^{+} f_{i m}
\end{aligned}
$$

where $\varepsilon_{f m}$ is the energy of the multiplet state $m$, $\varepsilon_{k \sigma}$ that of an unperturbed conduction state of wavevector $k$ and $\operatorname{spin} \sigma, N_{\ell}$ the total number of lattice sites, $c_{k \sigma}, c_{k \sigma}^{+}$the Fermion operators for conduction states, oV a volume deformation, $B(V)$ the Bulk lodulus 
and $e$ a deformation potential for state $n$, $H$ an applied nagnetic field and $b_{J}$ the lande factor for the nultiplet.

\section{Mean-Field Theory for the One-Flectron Green's Functions}

We are now in a position to use the Equation-of-liotion method ${ }^{2}$ to calculate the single electron Green's Functions in lowest orier. While this may appear to be a very crude solution to the problem (analogous to the "Hubbard I"-type approxinations used for transition metals ${ }^{3}$ ), it has certain interesting features worth noting on the way to a better solution. It gives a natural generalization for the lattice case of the known lowest-order results for the single impurity problem, such as those of Varma and Yafet or Ramakrishnan. 5 It exhibits a renormalization of the bare $f$-level similar to that obtained by Haldane 6 using rather general scaling arguments. One also obtains, almost trivially, results derived for the spin $1 / 2 \mathrm{f}-\mathrm{level}$ case using diagrammatic techniques, and is easily generalized to the full multiplet case. The basic idea is to decouple higher order Green's functions arising from the non-trivial anticommutation rules (Eqs. (1.4)) back down to lowest order. Physically, this corresponds to imbedding each $f$ electron shell in a mean lattice; thus, it is basically a mean-field type of theory. We start by defining operators for propagating excitations on the lattice, analogous to the $\mathrm{C}_{k \sigma}, \mathrm{C}_{k \sigma}^{+}$:

$$
f_{k m}^{+}=\mathrm{N}_{\ell}^{-1 / 2} \sum_{i} f_{i m}^{+} e^{-i \vec{k} \cdot \vec{r}_{i}} \text {, etc. }
$$

One may atiempt to write down a ground-state Ferni liquid sype wavefunction using these cperators but it turns out to be very hard to evaluate because of the non-trivial anticomatation rules for the f-operators. The Grean's function method, on the other hand, avoids the necessity of having to consider wavefunctions at all. Let us consider the usual (retarded) thermal Green's functions 8

$$
\mathrm{G}^{\mathrm{AB}}(\omega) \equiv\langle\mathrm{A} ; \mathrm{B}\rangle \omega
$$

and let us choose $A, B$ to be $f_{k m}$ or $C_{k \sigma}$ and $f_{k m}^{+}$or $C_{k \sigma}^{+}$, respectively. Their equations of motion are given by

$$
\begin{aligned}
\omega G_{k}^{m m^{\prime}}(\omega) & =\left\langle\left[f_{\mathrm{km}}, f_{\mathrm{km}}^{+}\right]_{+}\right\rangle \\
& \left.+\ll\left[f_{\mathrm{km}}, \mathrm{H}\right] ; \mathrm{f}_{\mathrm{km}}^{+},\right\rangle_{\omega},
\end{aligned}
$$

with similar equations for the other one-particle Green's functions. For simplicity, let us consider first the case where we have a simple spin-1/2 f-doublet so $\mathrm{m}$ can be replaced by a spin index 0 . 
By Eqs. (1.4), (1.5) we have

$$
\begin{aligned}
& {\left[f_{k \sigma^{\prime}}, H\right]=\left(\varepsilon_{f}-g \mu_{B} H-e\left\langle n^{f}\right\rangle / B(V)\right) f_{k \sigma}} \\
& \quad+N_{\ell}^{-1 / 2} \sum_{j k^{\prime} \sigma^{\prime}} e^{i\left(\vec{k}-k^{\prime}\right) \cdot r_{j}} v_{k^{\prime} \sigma^{\prime}} Q_{\sigma \sigma^{\prime}}^{j} c_{k^{\prime} \sigma^{\prime}},
\end{aligned}
$$

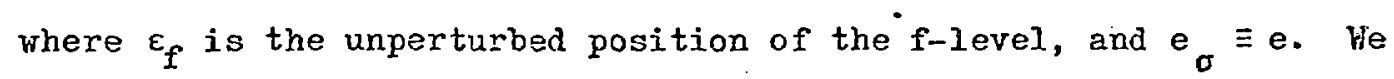
have eliminated of by minimizing the energy with respect to it. Using the representation $(1.4 \mathrm{c})$ for $Q_{\sigma \sigma^{\prime}}^{j}$ and decoupling the last product in $\mathrm{Eq} .(2.4)$, we obtain

$$
\left[f_{k \sigma}, H\right] \simeq \varepsilon_{f \sigma}^{*} f_{k \sigma}+v_{k \sigma}\langle Q \sigma\rangle c_{k \sigma},
$$

where

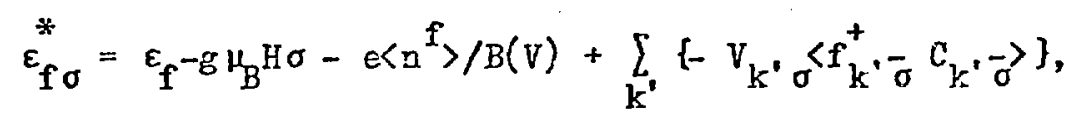

where $\bar{\sigma}$ denotes the spin opposite to $\sigma$ and

$$
\left\langle Q_{\sigma}\right\rangle=1-\frac{\left\langle n^{f}\right\rangle}{\sigma} \text {. }
$$

The equation of motion for the conduction electron pperator $C_{k \sigma}$ is rigorously

$$
\left[\mathrm{C}_{k \sigma}, \mathrm{H}\right]=\varepsilon_{\mathrm{k} \sigma \mathrm{k \sigma}} \mathrm{C}_{\mathrm{k} \sigma \mathrm{k} \sigma}+\mathrm{V}_{\mathrm{f}}
$$

Let $G_{k s \sigma}(\omega)$ stand for the matrix

$$
\left(\begin{array}{lll}
G_{k}^{f f}(\omega) & & G_{k}^{c f}(\omega) \\
G_{k}^{f c}(\omega) & & G_{k}^{c c}(\omega)
\end{array}\right)
$$

where $f$ refers to the $f-s p i n$ index and $c$ to the conduction electron spin index, both being $\sigma$ in this case. From Eqs. (2.3), (2.5) and $(2.6)$, we obtain

$$
G_{k \sigma}^{-1}(\omega)=\left(\begin{array}{cc}
\frac{\omega-\varepsilon_{f \sigma}^{*}}{\left\langle Q \frac{\partial}{\zeta}\right.} & v_{k \sigma} \\
v_{k \sigma}^{*} & \omega-\varepsilon_{k \sigma}
\end{array}\right) \text {. }
$$


Thus the poles of the one-electron Green's function are at

$$
\begin{aligned}
& E_{k \sigma}^{ \pm} \equiv \frac{1}{2}\left(\varepsilon_{f \sigma}^{*}+\varepsilon_{k \sigma} \pm 4_{k \sigma}\right) ; \\
& s_{k \sigma} \equiv\left[\left(\varepsilon_{f \sigma}^{*}-\varepsilon_{k \sigma}\right)^{2}+4\left|v_{k \sigma}\right|^{2}<Q_{\sigma}\right]^{1 / 2}
\end{aligned}
$$

which would correspond to one-electron hybridized "bands" for the non-interacting case (i.e., for $f_{k \sigma}, f_{k \sigma}^{+}$obeying ordinary fermion statistics) if $\varepsilon_{f \sigma}^{*}$ was replaced by $\varepsilon_{f}$ and $\left\langle Q_{\sigma}\right\rangle$ by 1 . There is a "hybridization gap" of order $\pi|V|^{2}<Q / \%$ ( $W$ is the conduction band width), which is, horever, an indirec: (zone-boundary) gap (see Fig1). From $\mathrm{Eq} \cdot(2.9)$ it is obvious that the self-energy matrix $\sum_{\sim} k \sigma(\omega)$ (defined as $\mathrm{G}_{k \sigma}^{(0)-1}(\omega)-\mathrm{G}_{\mathrm{k} \sigma}^{-1}(\omega)$, where $\mathrm{G}_{k \sigma}^{(0)}(\omega)$ is the Green's function matrix for the non-interacting case) is real for all $w$, i.e., Fermi-liquid-like behavior has been "built in" the nodel from the outset by the very nature of the decoupling approximation. The themodynamic averages of the felectron occupation, etc. can be obtained from the general relations of the form 8

$$
\langle A B\rangle_{T}=\frac{1}{\pi} \int_{-\infty}^{+\infty} d \omega f(\omega-\mu) \underset{n \rightarrow 0^{+}}{\operatorname{Lim}} \operatorname{Im}\left\langle B_{;} A\right\rangle_{\omega+\text { in }}
$$

where $A, B$ are any one-electron operators, $f(\omega-\mu)$ is the Ferrai function, and $\mu$ the chemical potential. The explicit expressions in the case of our model are given in Rer. (9). The chenical potential $\mu$ is determined by the condition

$$
\sum_{\sigma}\left\{\left\langle n_{\sigma}^{f}\right\rangle+\left\langle n_{\sigma}^{c}\right\rangle\right\}=n_{e} \text {; }
$$

$\mathrm{n}$ being the given number of electrons par unit cell. Eqs. (2.6), $(2.9),(2.11)$ and $(2.12)$ must be solved self-consistently at any terperature and applied field $H$ for given $\varepsilon_{f}, V_{k \sigma}, \varepsilon_{k \sigma^{*}}$ The selfconsistent determination of the chemical potential $\mu$ is, of course, a new feature not present in the impurity case.

From the results of such calculations we may obtain quantities such as the f-occupation, susceptibility, electronic specific heat and unit cell volume as functions of the position of the f-level, temperature, etc. Such numerical calculations were carried out for simple model systems and reported in Pef. 9 (although lattice effects were ignored there). We gain further insight into our model by neglecting the $k$-dependence of the hjbridization matrix elements and assume the unperturbed conduction band is characterized by a constant density of states $\alpha=1 / \mathrm{N}$ per spin). 
Let us define an effective density of f-states $\tilde{n}_{\sigma}(w)$ such that

$$
\left\langle n_{\sigma}^{f}\right\rangle=\int_{-\infty}^{+\infty} \tilde{n}_{\sigma}^{f}(\omega) f(\omega-\mu) d \omega ;
$$

and let $n_{\sigma}^{c}(\omega)$ be the conduction electron density of states (DOS).

From Eq. (2.11) it may be shown that

$$
\begin{array}{ll}
n_{\sigma}^{c}(\omega)=\rho ; \tilde{n}_{\sigma}^{f}(\omega)=V^{2}<Q>^{2} \rho /\left(\omega-\varepsilon_{f}^{*}\right)^{2} & \text { for } \omega \text { outside the gap } \Delta \\
n_{\sigma}^{c}(\omega)=n_{\sigma}^{f}(\omega) .=0 & \text { for } \omega \text { inside the gap } \Delta .
\end{array}
$$

with

$$
\Delta=4 V^{2}\left\langle Q_{\sigma}\right\rangle \rho,
$$

and where

$$
\left\langle Q_{\sigma}\right\rangle=1-\langle n \stackrel{f}{\sigma}\rangle=1-\frac{1}{2}\langle n \stackrel{f}{ }\rangle
$$

for the paramagnetic case with zero apolied field. The effective density of one-electron-like excitations is shown in Fig. 2. A peculiarity (compared so conventional one-electron DOS) is that the total number of available states is given by

$$
\sum_{\sigma} \int_{-\infty}^{\infty}\left[\tilde{n}_{\sigma}^{f}(\omega)+n_{\sigma}^{c}(\omega)\right] d \omega=2\left[1+\left\langle Q_{\sigma}\right\rangle\right]=4-\left\langle n^{f}\right\rangle
$$

so that the maximur filling of these 4 hybridized bands (2 per spin) is with 3 electrons, not 4 , as required by the correlation restriction of only one f-electron per site. It may also be shown that the total number of states in the lower band $\mathrm{K}$ - satisfies $1<\mathrm{K}-22$. Thus, at $1=0$, for one electron per unit cell, $\mu$ must always lie in the lower band; whereas for two electrons per unit cell, $\mu$ nust lie in the upper band. This result was also obtained by Roberts and Stevens"7. For the case of 2 electrons/cell, in the non-interacting case, $\mu$ would always lie in the gap. Thus the present result implies a violation of Luttinger's Theorem ${ }^{10}$ which states that the Fermi surface volume in the interacting case at $\mathrm{T}=0$ should be identical to that in the non-interacting case, as has been emphasized by Hartin ${ }^{11}$. In the present model it may be shown for the paranagnetic case that

$$
V_{F S}=v_{F S}^{(0)}+\frac{2 \pi^{3}}{\Omega_{a}} \frac{\left\langle n^{f}\right\rangle^{2}}{\left[1-1 / 2\left\langle n^{f}\right\rangle\right]}
$$


where $V_{F S}$ is the volume inside the Fersi surface, $V_{F S}^{(0)}$ that in the non-interacting case, $\Omega_{a}$ the unit cell volue and $\langle n\rangle$ the average number of $f$-electrons per site. In the case where $\left\langle n^{f}\right\rangle=1$, this would yield a difference corresponding to one election exactly between $V_{F S}$ and $V_{F S}^{(0)}$. In the full nuitiplet case the discrepancy between $V_{F S}$ and $V_{F S}^{(0)}$ vanishes as $1 / \mu$, horever. Evaluation of $\varepsilon_{f \sigma}^{*}$ defined in $\mathrm{Eq} \cdot(2.6)$, gives using $\mathrm{Eq} \cdot(2.11)$

$$
\stackrel{\because}{\varepsilon_{f \sigma}}=\varepsilon_{f}-g \mu_{B} H \sigma-\frac{e\langle n f\rangle}{B(V)}+v^{2} \alpha Q \ln \left|\frac{W}{\varepsilon_{f \sigma}-\mu}\right| .
$$

The second and third terns are merely the Zeeman and deformation potentjal terms respectively, while the last tern yields a Iogarithmic upward renormalization of the f-level which has been found for the single-impurity problem previously 4,5 and is similar to tine general scaling result of Haldane $e^{6}$ for the single impurity.

The generalization of the above theory to the full $\mathrm{N}$-fold multiplet case is quite straight forverd, but will not be givan here for reasons of space. The main point is that, as stated above, $\left\langle Q_{m m}\right\rangle$ becomes $O\left(\frac{1}{N}\right)$. The hybridization of the multiplet with a single conduction hand produces $(H+2)$ hybridized "bands", and the hybridization splitting of these bands is reduced by a factor of $O\left(\frac{1}{N}\right)$. The f-levels thus can renormalize up towards the Fermi level or even above it.

While calculations for quantities such as the f-occupation, susceptibility, resistivity, etc. from the above model are qualitatively in agreement with experiment (at least in the strongly mixed-valence regine where $\left.\varepsilon_{f}^{*}+\mu\right)$, a rigorous justification for the above simple mean-field decoupling theory (e.g., from scaling ideas or other methods) is lacking. It is interesting to note, however, that for large $N$ the above results becoze almost identical to those derived by Read and Newns 12 by completely different methods. Our present feeling is that there is some rerit to carrying out detailed numerical calculations for a real (as opposed to a model) systen with the full multiplet structure included, in the case of large $\mathrm{N}$ and in the strongly mixed valence limit. This has not as yet been done. In the Kondo regine, however, the above type of theory is clearly not good enough, and we shall discuss improvements to it in Section 4. 

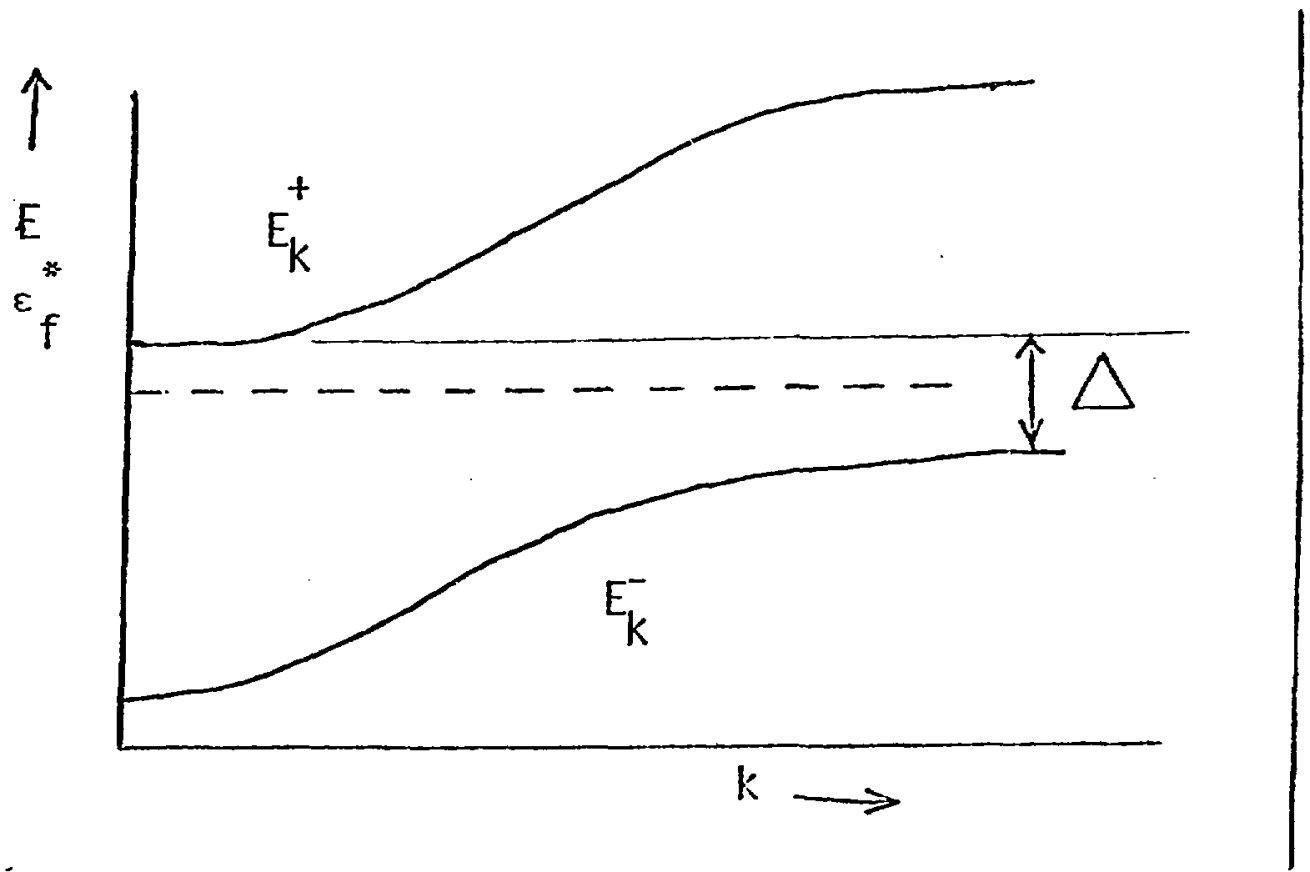

Fig. 1. Schematic of k-dependence of positions of poles of the oneelectron creen's function from the mean-field theory.

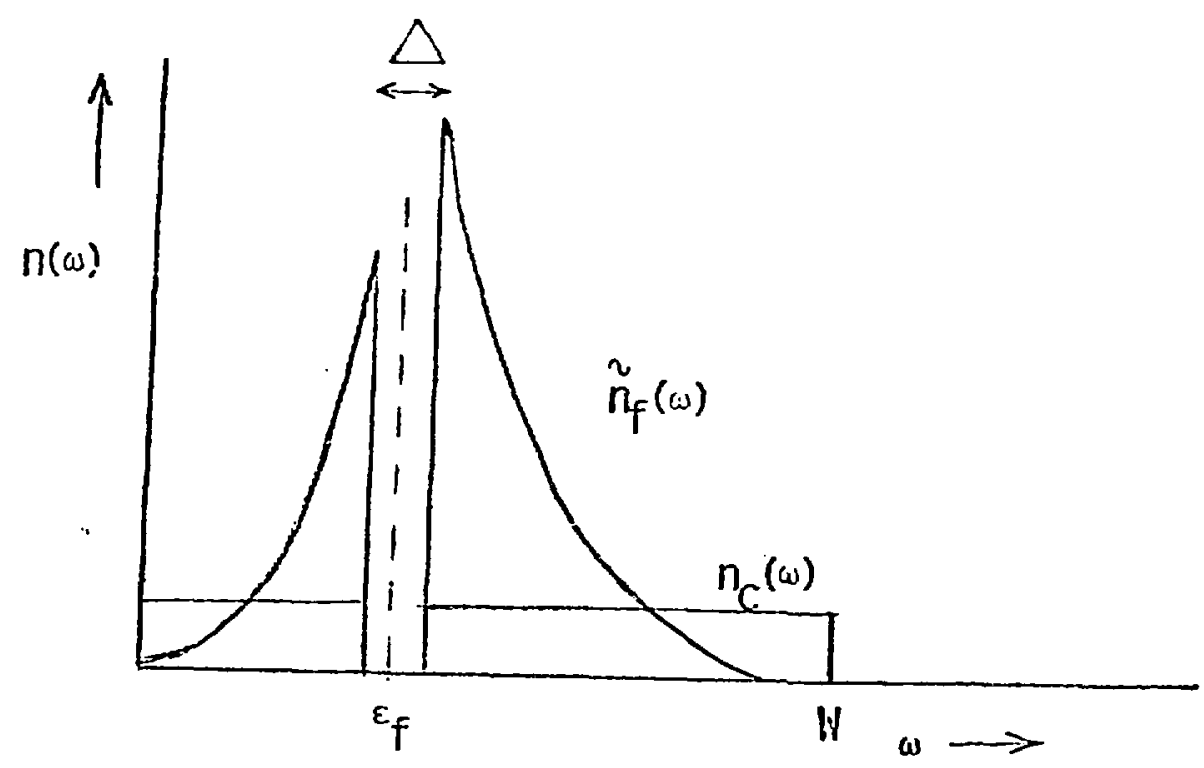

Fig. 2. Effective densities of "f-like" and conduction-like states for our simple model obtained from the nean-field theory. 


\section{The Two-Particle Green's Function and the Dynamical Susceptibility}

As is well-known ${ }^{13}$, the magnetic scattering of neutrons from a crystal is givail by the scattering function

$$
S(\vec{Q}, \omega)=f^{2}(\vec{Q})\left[1-e^{-\beta \omega}-1 \sum_{\alpha \beta}\left(\delta_{\alpha \beta}-\hat{Q}_{\alpha \beta} \hat{Q}_{\beta}\right) \operatorname{Im} x^{\alpha \beta}(\vec{q}, \omega)\right.
$$

where $\vec{Q}$ is the wavevector transfer of the neutron, hw its energy loss, $\hat{Q}$ is a unit vector along $Q, \beta$ is $1 / x T, f(d)$ is the magnetic form factor for the magnetic ions, and $\chi^{\alpha \beta}\left(q_{q}^{+}, \omega\right)$ is the dynamical susceptibility at the reduced wavevector $\vec{q}$ and frequency $\omega_{0}$. Iet us consider only scattering by the f-electrons for our simple model system of spin $-1 / 2 \mathrm{f}$-doublets. In the paramagnetic case, we need only consider $x^{+}(\vec{q}, \omega)$, which may be related to the 2-particle Green's function by

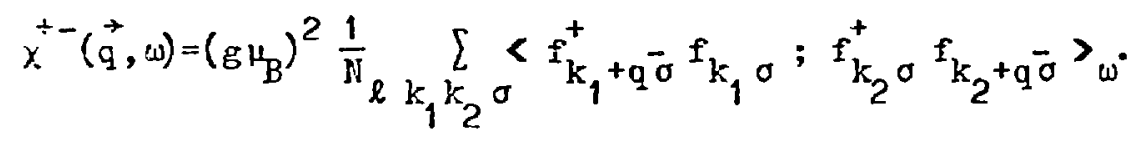

We evaluate the Green's functions by the Equation-of-Motion Hethod. Let us consider the operators

$$
\begin{aligned}
& \mathrm{Ni}_{1}^{+}(k \sigma, q) \equiv f_{k+q \bar{\sigma}}^{+} \cdot f_{k \sigma} ; \mathrm{N}_{2}^{+}(k \sigma, q) \equiv c_{k+q \bar{\sigma}}^{+} \cdot f_{k \sigma} \\
& \mathrm{H}_{3}^{+}(k \sigma, q) \equiv f_{k+q \bar{\sigma}}^{+} \cdot c_{k \sigma} ; \mathrm{NH}_{4}^{+}(k \sigma, q) \equiv \mathrm{c}_{k+q \bar{\sigma}}^{+} \cdot c_{k \sigma} \cdot
\end{aligned}
$$

Define the general Green's function

$$
G^{i j} \quad\left(\omega k^{\prime} \sigma \sigma^{\prime} q\right) \equiv\left\langle\stackrel{H}{i}_{i}(k \sigma, q) ; N_{j}\left(k^{\prime} \sigma^{\prime}, q\right)\right\rangle_{\omega} \text {. }
$$

In evaluating $\left[\mathrm{N}_{i}^{+}(k \sigma, q), H\right]$ we ray use the definitions $(1.1)$ to treat products of many $f$ operators on a single site exactly, but on different sites, we decouple four-operator products back down to two in all possible ways. We use the comatation relations given by Eqs. $(1.4)$, and obtain

$$
\begin{aligned}
& {\left[N_{1}^{*}(k \sigma, q), H\right] \simeq\left(\varepsilon_{f \sigma}-\varepsilon_{f \sigma}\right) N_{1}^{+}(k \sigma, q)+v_{k+q}\left\langle Q \sigma N_{2}^{+}(k \sigma, q)\right.} \\
& -v_{k}<Q=N_{3}^{+}(k \sigma, q)+\left\langle f_{k \sigma \sigma^{+} f^{+}}^{+}\right\rangle \frac{1}{N_{\ell}} \sum_{K} v_{K+q} N_{2}^{+}(K \sigma, q) \\
& -\left\langle\mathrm{f}_{\mathrm{k}+\mathrm{q} \sigma}^{+}-\mathrm{f}_{\mathrm{k}+\mathrm{q} \sigma}-\frac{1}{\mathrm{H}_{\ell}} \sum_{K} \mathrm{~V}_{\mathrm{K}}^{\mathrm{N}_{3}^{+}}(\mathrm{K} \sigma, \mathrm{q})\right.
\end{aligned}
$$

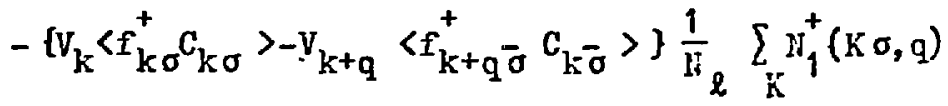


with similar equations for the commutators of $\mathrm{H}$ with $\mathrm{N}_{2}^{+}, \mathrm{N}_{3}^{+}, \mathrm{H}_{4}^{+}$. The last three terms in $\mathrm{Eq} .(3.5)$ arise from the kinematic restrictions to single occupation of the f-sites built into the f-operators, which ve may term a "blocking effect". Substitution of Eq. (3.5) into the equations of notion leads to a set of four coupled integral equations for the $\mathrm{G}_{\mathrm{kk}}^{j \mathrm{j}} \sigma \sigma^{\circ} \mathrm{q}(\omega)$, which can hovever be solved, since by Eq. (3.2) we need only $\underset{\mathrm{kk}^{\prime} \sigma}{\sum} G_{\mathrm{kk}^{\prime} \text { ooq }}^{11}(\omega)$. The solutions for $\chi^{+-}(\vec{q}, \omega)$ has the following structure

$$
x^{+-}(\vec{q}, \omega)=\left[\{1+\pi(\vec{q}, \omega)\}^{-1} \cdot z(\vec{q}, \omega)\right]_{1}
$$

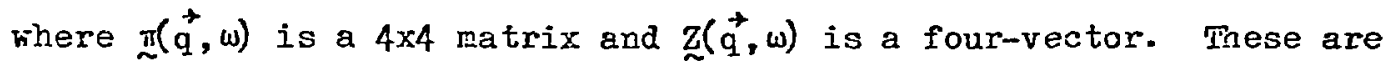
functions of $\left\langle n_{k \sigma}^{f}\right\rangle,\left\langle n_{k+q}^{f}\right\rangle,\left\langle n_{k}^{c}\right\rangle,\left[\omega+E_{k \sigma}^{ \pm}-E_{k+q}^{ \pm}\right]^{-1}$, etc.

$\mathrm{Z}_{1}(\stackrel{+}{\mathrm{a}}, \omega)$ is analogous to a single non-interacting band susceptibility ior this tro-band model, so that $\pi$ gives an effective enhancenent. Tine numerical calculations for $S(\tilde{Q}, \omega)$ are shom in Fig. 3 , for $a$ nocel fcc lattice of ions containing both $f$ and conduction orbitals (with only nearest neighbor hopping intesrals). The occupation numbers and bands were calculated from the self-consistent one electron theory described in the last section. Two points should be noted. First, the enhancement effect due to the f-electron correlations is appreciable only in the dynamic regime $\omega<\Delta$, the hybridization gap $\left(\Delta \sim \pi v^{2} \rho\right)$. Secondly, in the strongly mixedvalence rezine, $S(Q, \omega)$ exhibits a central peak and a peak at $\omega \sim \Delta$. The latter peak is a maximum when $q$ is at the zone-boundary and decreases to zero intensity as $q+0$, although it is essentially dispersionless. This is consistent with the gap being an indirect one, as may be seen from Fig. 1. This peak also broadens and decreases in intensity as $(\mathrm{kT} / \Delta)$ increases. It is interesting to note that such qualitative behaiior was found for the peak observed at $10 \mathrm{meV}$ energy transfer in the inelastic neutron scattering from single-crystal paramagnetic TuSe by Grier and Shapiro14, although the simple two band model presented here is certainly a very crude representation of the nore conplex "band structure" in a real nultiplet system. Finally, the central peak around $\omega=0$ has a width which is fairly temperature insensitive, in agreement with neutron scattering experiments on mixed-valent systers 15 .

4. Incorporation of Fluctuations Effects in the One-Electron Green's Function

We may extend the theory given in Section 2 by using a higherorder decoupling procedure. Using $\mathrm{Eq} .(1.4 \mathrm{c})$, we may write $\mathrm{Eq}$. $(2.4)$ as

$$
\left[f_{k \sigma}, H\right]=\varepsilon_{f \sigma}^{*} f_{k \sigma}+v_{k} c_{k \sigma}-\frac{1}{N} \sum_{\ell j, k_{1}} v_{k_{1}} e^{-i\left(\vec{k}_{k}-\vec{k}_{1}\right) \cdot \vec{r}_{j}}\left[z_{j k_{1}}^{\sigma}-s_{j k_{1}}^{\sigma}\right] \text {, }
$$




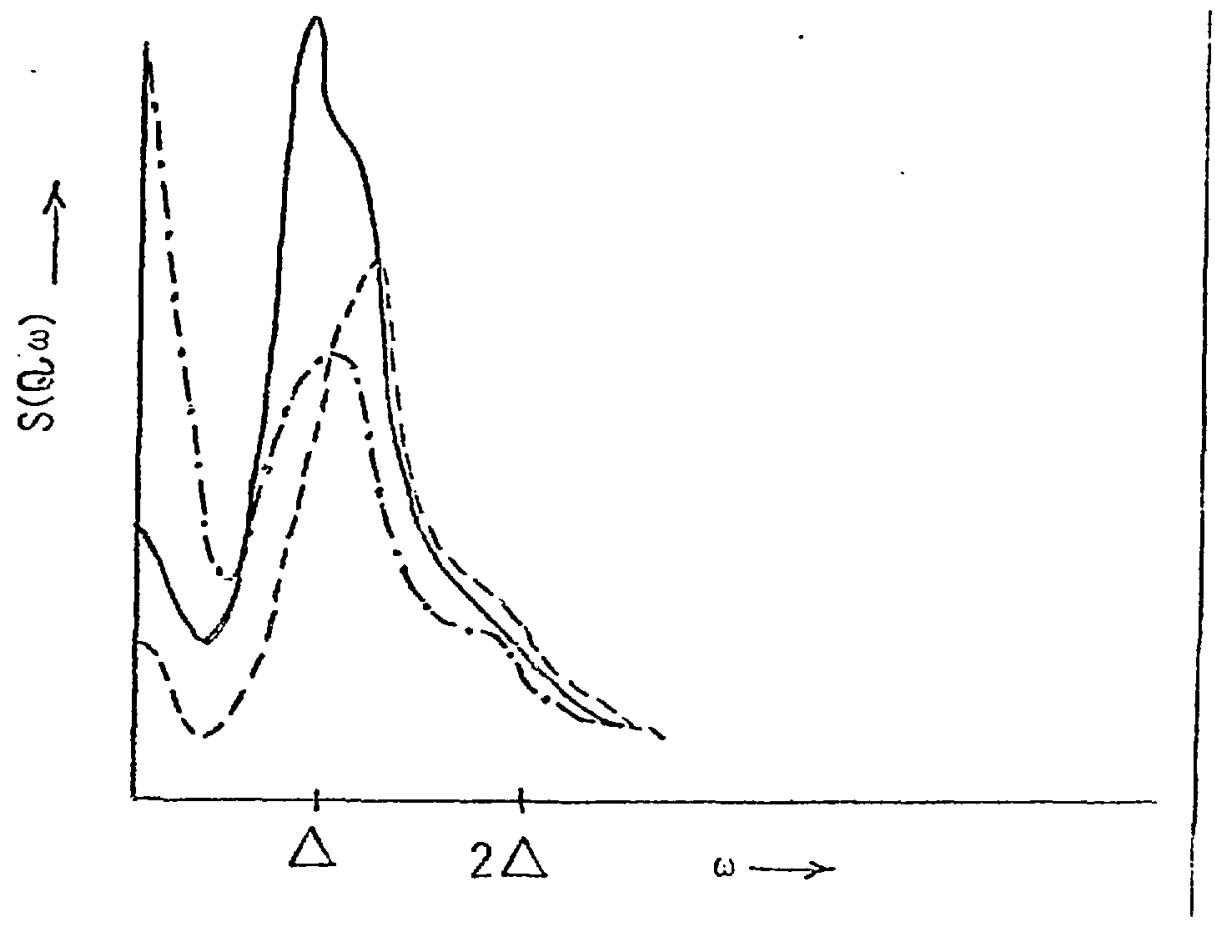

Fis. 3. $S(Q, \omega)$ for $Q$ at the point $2 \pi / a(1,0,0)$ for the model fcc Anderson lattice discussed in the text. The full curve denotes the calculation with the enhancerent factcr and the dashed curve the unenhanced $S(Q, \omega)$ for $\mathrm{kT} / \Delta=0.18$. The. dot-ash curve shows the enhanced $S(Q, \omega)$ for $\mathrm{kT} / \Delta=1.76$.

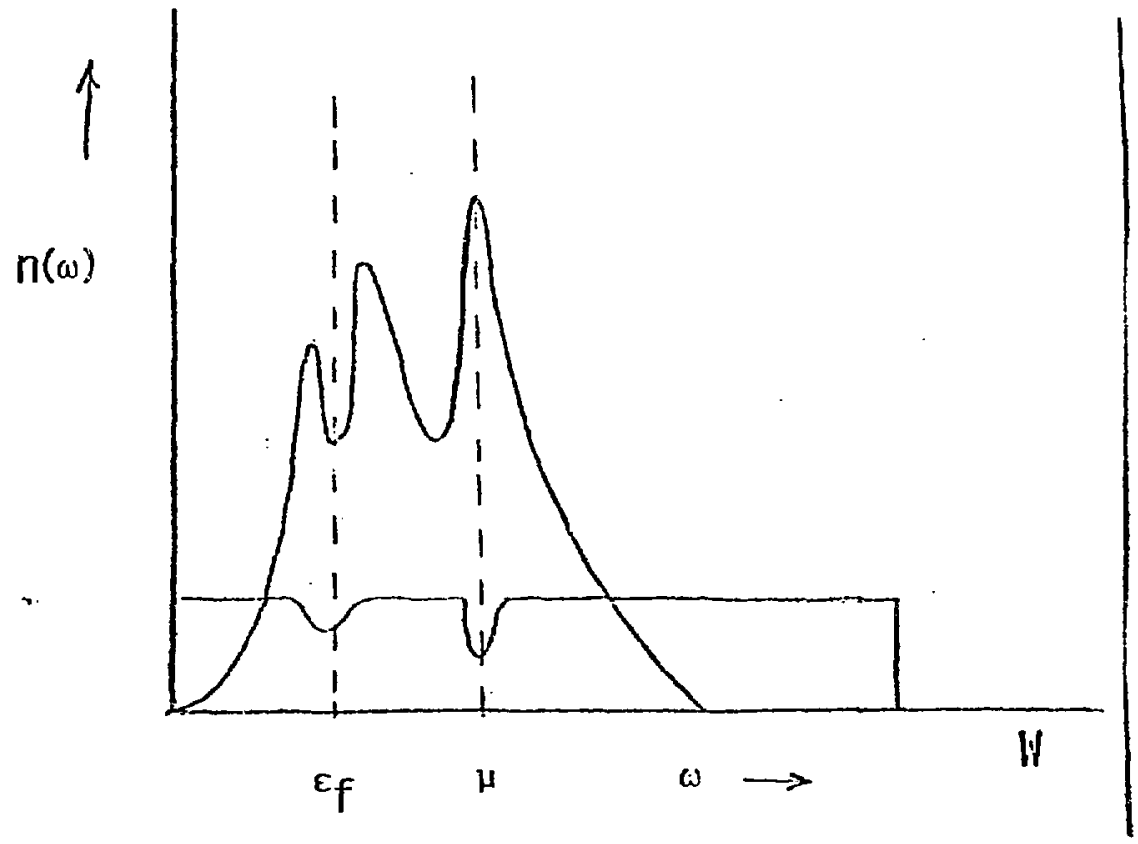

Fig. 4. Effective densities of "f-like" and conduction-like states for an Anderson lattice with $\varepsilon_{f}$ well below the Fermi-level from the one-electron Green's Iunction including spin and charge fluctuation effects. 
where we have assuned that $\mathrm{V}_{\mathrm{k} \alpha}$ is independent of $\sigma$. The operators $\mathrm{Z}$ and $\mathbf{S}$ are defined as

$$
z_{j k_{1}}^{\sigma} \equiv f_{j \bar{\sigma} j^{-}{ }^{+}}^{C_{k_{1} \sigma}} ; S_{j k_{1}}^{\sigma} \equiv f_{j \bar{\sigma}}^{+} f_{j \sigma_{k_{1} \sigma}}^{C}
$$

The equations of notion which result from Eqs. (2.8) and (4.1) yield Green's functions involving $Z$ and $S$. The procedure is to take next the equations of motion of these Green's functions and decouple operators which involve f-operators or c-operators on different sites, except those that occur in the combinations given in $\mathrm{E}_{1}$. (4.2). In this way, we keep the effects of spin and charge fluctuations on an individual $\mathrm{f}$-shell explicitly in the problem. The method is similar to that used by $\mathrm{Nagaoka}^{16}$ in his treatment of the single Kondo impurity, althougi. in that case, charge fluctuations (given by the z-operators cannot occur). The next result of this procedure is that Eq. (2.9) in the mean-field theory is replaced by

$$
G_{j k \sigma}^{-1}(\omega)=\left(\begin{array}{cc}
\left.\frac{\omega-\varepsilon_{f \sigma}^{*}(\omega)}{\langle Q}(\omega)\right\rangle & v_{k} \\
v_{k}^{*} & \omega-\varepsilon_{k_{\sigma}}
\end{array}\right)
$$

i.e., the quantities $\varepsilon_{f \sigma}^{*}$ and $\left\langle Q_{\sigma}\right\rangle$ in the mean-field theory now become complex and frequency-dependent. The explicit expressions for $\varepsilon_{f}^{*}(\omega)$ and $\langle Q(\omega)\rangle$ are $e^{17}$

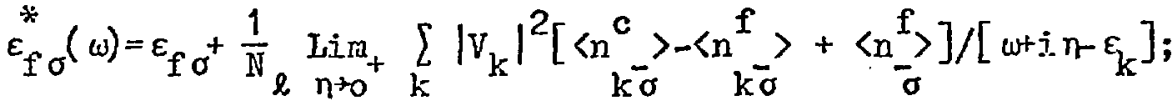

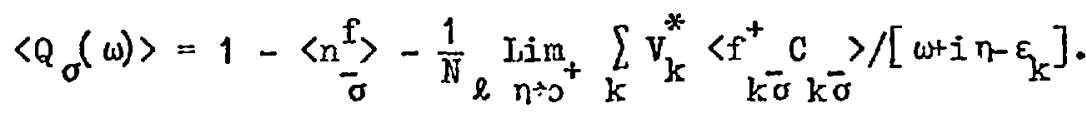

The expectation values $\left\langle\mathrm{n}_{\mathrm{k}}^{f}\right\rangle$, etc. are still given by Eqs. (2.11), e.g. ,

$$
\left\langle n_{k \sigma}^{f}\right\rangle=\frac{1}{\pi} \int_{-\infty}^{+\infty} d \omega f(\omega-\mu) \operatorname{Im} G_{k \sigma}^{f f}(\omega)
$$

etc., and the self-consistent solutions of Eqs. (4.3) to (4.5), together with the condition (2.12) for the chemical potential leads to a set of coupled integral equations for $\varepsilon_{f \sigma}^{*}(\omega)$ and $\left\langle Q_{\sigma}(\omega)\right\rangle$.

These integral equations are very hard to solve analytically, since they are highly singular as $\omega \rightarrow 0$ and $T \rightarrow 0$. We may, however, solve for $\varepsilon_{f}^{*}(\omega)$ in the limit where $\left|w_{1}\right|,\left|W_{2}\right| \gg k i \gg v$, where $V_{1}$ 
and $\mathrm{H}_{2}$ are respectively the lower and upper edges of the conduction band obtaining

$$
\varepsilon_{f}^{*}(\omega)=\varepsilon_{f}+V^{2} \rho\left[\phi_{1}(\omega)-\gamma\right]+i \pi f(\omega)+v^{2} \pi \psi\left(\omega, H_{1}, k T\right),
$$

where $\gamma$ is a irequency-independent constant, which must be determined self-consistently from Eqs. (4.4), and

$$
\psi\left(\omega, W_{1}, k T\right) \equiv \int_{W_{1}}^{\infty} d x \frac{f(x)}{x-\omega} .
$$

Here $f(x)$ is the Fermi function $\left[e^{x / x ?}+1\right]^{-1}$.

In the limit $\omega * 0$, the behavior of this solution is

$$
\varepsilon_{f}^{*}(0) \sim \varepsilon_{f}+\left|\varepsilon_{f}\right| \ln \left(T / T_{k}\right) \text {, }
$$

where

$$
\mathrm{T}_{\mathrm{k}}=\mathrm{T}_{\mathrm{o}} \mathrm{e}^{-\left|\varepsilon_{\mathrm{f}}\right| /\left(\mathrm{V}^{2} \rho\right)},
$$

with $T_{0} \sim \mathrm{H}$

This shows that even if the bare $\varepsilon_{f}$ is well below the Fermi level, its renormalization at $\omega=0$ (i..., s.t the Fermi level) can bring the effective $\varepsilon_{f}^{*}$ back up to the Ferai level, producirg a sharp temperature-dependent resonance at the Fermi level. It can then be shown that the $f$ DOS, $\tilde{n}_{f}(\omega)$, will have peaks both at $\omega \sim \varepsilon_{f}$ and in the vicinity of the Fermi level (see Fig. 4). This is the lattice analogue of the so-called "many-body resonance" or Abrikosov-Suhl resonance in the case of the single inpurity ${ }^{18}$. Similar results for the single impurity case have been derived by Lacroix and Cyrot 19 and for the lattice case by Lacroix 20 and by Raugartel and fiullerHartmann. 21 In order to see what happens in the more interesting low temperature region, the integral equetions will have to be solved (probably numerically). This has not yet been done. Equations (4.4) also have to be generalized to the full multiplet case, where in analogy with the single inpurity case, we expect the amplitude of the resonance at the Fermi level to be increased by a factor of $\mathrm{N}$. The existence of this resonance for $\mathrm{T} \lesssim \mathrm{T}_{\mathbf{k}}$ would be consistent with several experimental results for "deñse Kondo" systems (see for example, the lectures by Wilking $(22)$ at this School) which is the limit of our Anderson lattice as $\varepsilon_{f}$ sinks well below the Fermi level.

5. Correlation-Induced Pairing Instability

We may alsc use the Equation of rotion ilethod for the two- 
particle Green's functions to investigate whether the Anderson Iattice Hamiltonian (1.5) has a Cooper-pairing type instability, which is not driven by the usual phonon-mediated attraction. In order to show this, we study the dynarical response functions for pair creation. These are related to the two-particle Green's functions of the form $\ll \tilde{\mathbb{N}}_{i}^{+}(k, q) ; \tilde{\mathbb{N}}_{j}\left(k^{\prime}, q\right) \gg$, where

$$
\begin{aligned}
& \tilde{N}_{1}^{+}(k, q) \equiv f_{k+q}^{+} f_{-k+}^{+} ; \quad \tilde{N}_{2}^{+}(k, q) \equiv C_{k+q, t^{+}-k+}^{+} \\
& \tilde{N}_{3}^{+}(k, q) \equiv f_{k+q}^{+} \hat{t}^{+}-k+\quad ; \quad \tilde{N}_{4}^{+}(k, q) \equiv C_{k+q}^{+} \hat{t}^{+}-k+\cdot
\end{aligned}
$$

If these response functions diverge for $q, \omega \rightarrow 0$ at some $T=T$, it means that there is a spontaneous formation of Cooper pairs, i.e., a pairing instability. Using a decoupling procedure similar to that described in Section 3 for the calculation of the srin response function (where products of several f-operators on the same site were treated exactly but difierent sites decoupled) we obtain equations of motion of the following form:

$$
\begin{aligned}
& {\left[\tilde{\mathbb{N}}_{1}^{+}(k, q), H\right]=\left(\varepsilon_{f \sigma}+\varepsilon_{f \sigma}^{-}\right) \tilde{N}_{1}^{+}(k, q)+v_{k r q}\left\langle Q-Q_{\sigma}>\tilde{N}_{2}^{+}(k, q)\right.} \\
& +v_{k}\left\langle Q_{\sigma}>\tilde{N}_{3}^{+}(k, q)-\sum_{\sigma}\left(\varepsilon_{f \sigma}^{*}-\varepsilon_{f \sigma}\right) \frac{1}{N_{\ell}} \sum_{k_{i}} \tilde{i}_{1}^{+}\left(k_{1}, q\right)\right. \\
& +\left[\left\langle n_{k+q \sigma}^{f}\right\rangle+\left\langle n_{f-\sigma}^{f}\right\rangle-1-\left\langle f_{j \sigma^{f} j \sigma^{+}}^{f}\right] \frac{1}{N_{\ell}} \sum_{k_{1}} V_{k_{1+q}}\left(k_{1}, q\right) \tilde{I N}_{2}^{+}(k, q)\right. \\
& +\left[\left\langle n_{k+q \sigma}^{f}\right\rangle+\left\langle n_{k \bar{\sigma}}^{f}\right\rangle-1-\left\langle f_{j \sigma \sigma j \sigma}^{+f}\right\rangle\right] \frac{1}{\mathbb{N}_{\ell}} \sum_{k_{1}} v_{k_{1}} \tilde{N}_{3}^{+}\left(k_{1}, q\right) \text {. }
\end{aligned}
$$

By surming this equation over $k$, we may show that $\left\langle\frac{1}{n_{\ell}} \sum_{k_{1}} \tilde{H}_{1}^{+}\left(k_{1}, q\right)\right\rangle$ is always identically zero as it must be since it corresponds to $\left\langle f_{i}^{+} f_{i}^{+}\right\rangle$. Thus, the decoupling scheme does not violate the restriction that two f-electrons cannot be on the same site. Hence the fourth term on the right of Eq. (5.2) vanishes. Similar equations may be written down for the other operators in $\mathrm{Eq} .(5.1)$, and the solution of the resulting equations for the Green's function yields a vanishing denominator if the following equation is satisfied for $T=T_{C}$

$$
\begin{aligned}
& \frac{\langle Q\rangle}{N_{\ell k}} \sum_{k} \frac{2_{k}^{v_{k}^{2}} \varepsilon_{k}}{A_{k} \Delta_{k}}\left[\frac{1}{2 E_{k}^{+}} \operatorname{Tanh}\left(\frac{B_{c} E_{k}^{+}}{k}\right)-\frac{1}{2 E_{k}^{-}} \operatorname{Tanh}\left(\frac{\beta_{c} E_{k}^{-}}{2}\right)\right] \\
& \quad+[\langle Q\rangle-1] \frac{1}{N_{2}} \sum_{k} \frac{8 v_{k}^{2} E_{k}}{A_{k}\left(A_{k}^{2}-A_{k}^{2}\right)}=1 .
\end{aligned}
$$


where we have dropped the spin index, $B_{C} \equiv 1 / k_{B} T_{c}$,

$$
A_{k}=\varepsilon_{f}^{*}+\varepsilon_{k} \text {, }
$$

and $E_{k}^{t}, A_{k}$ are defined in Eqs. $(2 \cdot 10)$.

An exarination of the solutions of this equatica reveal the following features: (1) No solution for $\mathbb{T}_{c}$ exists if the Fermi Level $\mu$ lies in the lower band $E_{k}^{-}$(i.e., if $\varepsilon_{f}^{*}$ lies above $\mu$ ). This would be the case for a system with one electron per unit cell. (2) ${ }^{T}$ decreases rapidly as $\varepsilon_{f}^{*}$ noves dow below $\mu$. Thus it is oxily in the strongly mixed-valent regime that $T_{c}$ is largest. Insertion of typical values of $V$, conduction bandiidth $W$ and $\left(\varepsilon_{f^{-}}^{*} \mu\right)$ yield $T_{c}$ of the order of $1 \mathrm{~K}$ or less.

The primary insiability is in the response of $\left(\mathrm{C}_{\mathrm{k} t^{+}-\mathrm{f}^{-}}^{+} \mathrm{f}_{\mathrm{k} t^{+}-\mathrm{c}^{+}}^{+}\right)$, i.e., singlet pairing between f-electrons and conduction electrons. We ascribe this to an attractive interaction between these electrons of a form similar to the Schrieffer-Nolffe $f^{23}-\vec{J} \stackrel{\vec{\sigma}}{*} \cdot \vec{S}$ interaction in the Kondo regime and arising from the same physical origin. In the mixed-valence regine, however, there are f-electrons at the Fermi surfece, and thus the attractive interection leads to a pairing instability. The tendency towards instability with regard to singlet f-l pairing in mixed valence systers has also been discussed by Delienezies, 24 who, however, considers the usual phonon mechanism as the source of the attractive interaction. Our theory has not considered possible pair-breaking mechenisms such as the real scattering of f-electrons by spin or charge fluctuations. One can only appeal to the experimental observation that Fermi-liquid behavior is estimated in these systems at low temperatures. The fluctuation theory of Section 4 can in principle be incorporated into our formalism but this has as yet not been carried out. One might expect that, even in the Kondo regime, a sharp resonance of $f$-like states at the Fermi level might lead once again to significant $T_{c}$ 's due to the above mechanism. Further investigation of this pairing mechanism is necessary before one can attempt to identify it with what is observed in the recently discovered "heavy-fermicn" superconductors.25,26 
$*$

Work supported by the U.S. Departnent of Energy.

On leave frol Northern Illinois University, DeFalb, IL 60115.

Present address: Exxon Research and Engineering Company,

Corporate Research Iabs. Annandale, NJ 08801.

1. Hubbard, J., Proc. Roy. Soc. A285:542 (1965).

2. Miablikov, S. V., "Nethods in the Quantum Theory of Nagnetism" Plenum Press, New York (1967).

3. Hubbard, J., Proc. Roy. Soc. A276:238 (1963).

4. Varma, C. M. and Yafet, Y., Phys. Rev. B 13:2950 (1976).

5. Ramakrishnan, T. V., in: "Valence Fluctuations in Solids", L. H. Falicov, W. Hanke and M. B. Haple, ed., North-Holland, Amsterdam (1981).

6. Haldane, F. D. M., Phys. Rev. Lett. 40:416 (1978).

7. Roberts, H. and Stevens, K. W. H., J. Phys. C 13:5941 (1980).

8. Zubarev, D. N., USP. Miz. Nauk. 71:71 (1960).

9. Fedro, A. J. and Sinha, S. K., in: "Valence Fluctuations in Solids", L. M. Falicor, H. Hanke and M. B. Haple, eds., NorthHolland, Arnsterdam (1981) p. 329.

10. Inttinger, J. M., Phys. Rev. 119:1153 (1960).

11. Martin, R. H., Phys. Rev. Lett. 48:362 (1982).

12. Read, N. and Newns, D. H., to be published in these proceedings.

13. Izuyama, T., Kin, D. and Kubo, R., J. Phys. Soc. Japan 18:1025 $(1963)$.

14. Grier, B. H. and Snapiro, S. M., bid Ref. 11, p. 325; Phys. Rev. B 25:1457 (1982).

15. Lo ewenhaupt, M. and Holland-Montz, E., J. Appl. Phys. 50:754.6 (1979).

16. Nagaoka, Y., Phys. Rev A. 138:1112 (1965).

17. Fedro, A. J. and Sinha, S. K., Proc. Int. Conf. on Valence Instabilities, P. Wachter and H. Boppart, eds., Zurich, NorthHolland, Ansterdam (1982) p. 371.

18. Zlatic, V., Gruner, G. and Rivier, N., Solid State Comm. 14:139 (1974).

19. Iacroix, C. and Cyrot, M., Phys. Rev. B 20:1969 (1979).

20. Lacroix, C., J. Appl. Phys. $53(3): 2131$ (1979).

21. Baumgartel, H. and Huller-Hartmann, F., Proc. Int. Conf. on

Valence Instabilities, F. Wachter and H. Boppart, eds.,

Zurich, North-Holland, Amsterdan (1982) p. 57.

- 22. Wlikins, J. W., to be published in these proceedings.

23. Schrieffer, J. R. and Volff, P. A., Phys. Rev. 149:491 (1966).

24. De Menezies, 0. L. T., ibid, p. 53.

25. Steglich, F., Aarts, J., Bredl, C. D., lieke, W., Meschede, D., Franz, W. and Schäfer, H., Pnys. Rev. Lett. 43:1892 (1979).

26. Ott, H. R., Rudigier, H., Fisk, Z. and Sinith, J. L., Phys. Rev. Iett. 50:1595 (1983). 